\title{
PRENATAL DIAGNOSIS OF ORGANIC ACIDEMIAS AT A TERTIARY CENTER
}

\author{
Tanacan $\mathrm{A}^{1, *}$, Gurbuz $\mathrm{BB}^{2}$, Aydin $\mathrm{E}^{1}$, Erden $\mathrm{M}^{1}$, Coskun $\mathrm{T}^{2}$, Beksac $\mathrm{MS}^{1}$
}

*Corresponding Author: Dr. Atakan Tanacan, Department of Obstetrics and Gynecology, Division of Perinatology, Hacettepe University Hospital, Tip Fakültesi Street, Sthhiye, Ankara, Turkey. Tel: +90532-353-0892. Fax: +90-312-305-1910. E-mail: atakantanacan@yahoo.com

\begin{abstract}
The aim of this study was to share our experience in the prenatal diagnosis (PND) of organic acidemias (OAs) in our clinic. This study consisted of 10 cases in whom an invasive prenatal diagnostic test (IPNDT) was performed by a single physician for the PND of OAs. Median maternal age, parity, gestational week of IPNDT, prenatal test indications, OA types, method of IPNDT, IPNDT results and gestational outcomes were evaluated. Targeted mutation analysis was performed in fetal DNA for the specific mutations by using polymerase chain reaction (PCR) and direct Sanger sequencing. The diagnosis was confirmed by genetic targeted mutation analysis after birth. Median maternal age, parity and gestational week of IPNDT values were 30 (range 21-35), one (range 0-4) and 11.5 (range 11-17), respectively. Indications for IPNDT were mother being a carrier of the disease for one case $(10.0 \%)$ and at least one child with $\mathrm{OA}$ in the family for nine cases $(90.0 \%)$. Organic acidemia types investigated were maple syrup urine disease (MSUD), methylmalonic acidemia (MMA) and isovaleric acidemia (IVA) in five $(50.0 \%)$, three $(30.0 \%)$ and two $(20.0 \%)$ patients, respectively. Chorion villus sampling (CVS) was done in seven (70.0\%) patients and amniocentesis was performed in three (30.0\%) patients. Eight fetuses $(80.0 \%)$ were found to be healthy and two fetuses (20.0\%) were found to be affected (one case with IVA and one case with MMA). The two
\end{abstract}

\footnotetext{
${ }^{1}$ Department of Obstetrics and Gynecology, Division of Perinatology, Hacettepe University Hospital, Ankara, Turkey

${ }^{2}$ Department of Pediatrics, Division of Pediatric Metabolism, Hacettepe University Hospital, Ankara, Turkey
}

pregnancies $(20.0 \%)$ with affected fetuses were terminated. Prenatal diagnosis of OAs is critical. Appropriate prenatal counseling should be given to families with known risk factors.

Keywords: Invasive prenatal diagnostic test (IPNDT); Organic acidemia (OA); Prenatal diagnosis (PND); Pregnancy outcome.

\section{INTRODUCTION}

Organic acidemias (OAs) are a group of rare hereditary disorders characterized by increased excretion of organic acids in urine [1]. Deficiencies of specific enzymes in the breakdown pathways of amino acids lead to elevated levels of organic acids in the organism, causing alterations in several systems, mostly the central nervous system [1].

The incidence of OAs ranges between 3.7-12.6/ 100,000 according to various studies $[2,3]$. On the other hand, a much higher frequency was reported in a study that consisted of a preselected high-risk group of patients [4].

The most common types of OAs are propionic acidemia (PA), methyl malonic acidemia (MMA), branched chain organic acidemia [which includes isovaleric aciduria (IVA)] glutaric acidemia Type I, and multiple carboxylase deficiency (MCD) [5]. Maple syrup urine disease (MSUD), which occurs due to elevated branched chain amino acids, is also regarded as a branched chain amino acidemia [5]. All OAs are autosomal recessively inherited disorders [5].

Generally, signs and symptoms of these disorders develop during the newborn period or early infancy. Following an initial period of well-being, patients experience a life-threatening episode of metabolic acidosis with increased anion gap. These episodes may be associated with significant morbidity and even mortality [6]. Diagnosis of OAs can be challenging in newborns as the presenting 
symptoms may be mistaken for other conditions such as neonatal sepsis [7]. Although parental consanguinity may arouse suspicion for the diagnosis of OAs, definitive diagnosis usually cannot be anticipated without index cases. Use of gas chromatograph-mass spectrometry (GC-MS) and tandem MS has facilitated the diagnosis of OAs in the past few decades [8]. Diagnosing OAs within the first 24-48 hours of life is critical as appropriate management protocols may prevent serious morbidity and even mortality [5]. Thus, neonatal screening programs have vital importance, especially in countries with higher rates for OAs $[9,10]$.

Another important issue is providing suitable genetic counseling for couples with a history of OAs in their families, as prenatal diagnosis (PND) is available for most of them. Assessment of certain metabolites in the amniotic fluid, analysis of enzyme activities in amniocytes/chorionic villi and targeted mutation analysis may all be performed for PND of OAs [11,12]. Prenatal diagnosis is crucial as it may give parents the opportunity for termination of pregnancy in severe cases and may give the physicians enough time for referral of the patients to tertiary healthcare centers. The aim of this study was to share our experience in the PND of OAs in our clinic.

\section{MATERIALS AND METHODS}

This study consisted of 10 cases on whom invasive prenatal diagnostic test (IPNDT) was performed by a single physician for PND of OAs at the Division of Perinatology, Department of Obstetrics and Gynecology, Hacettepe University Hospital, Ankara, Turkey, between January 1, 2000 and December 31, 2017. The required data was retrospectively obtained from the electronic database of Hacettepe University.

Prenatal diagnosis was performed on couples with mothers being carriers of the disease or having at least one child with OA in their families. Patients gave written informed consent and their approval was obtained prior to the invasive procedures. All pregnancies were evaluated by the Department of Genetics and Clinical Biochemistry, Hacettepe University Hospital, Ankara, Turkey, within the framework of the PND program. Genomic DNA was obtained from venous (whole) blood of the index cases and the parents. The blood samples were collected in EDTA $\mathrm{K} 2$-containing vacutainers and transferred to the laboratory for targeted mutation analysis.

With the known genotypes of the index cases and parents before pregnancy, pregnant women were referred to the Division of Perinatology, Hacettepe University Hospital, Ankara, Turkey, for PND. Chorionic villus sampling (CVS) was performed between the 11th to 14th gestational weeks (all CVS procedures were performed by the transabdominal route) and amniocentesis (AC) was performed between the 16 th to 20 th gestational weeks. All women at risk of $\mathrm{Rh}$ isoimmunization received 300 $\mu \mathrm{g}$ of $\mathrm{Rh}-\mathrm{Ig}$ following the invasive procedures. Genomic DNA was extracted from chorionic villi and amniotic fluid samples using the DNA extraction kit (Gentra Systems, Minneapolis, MN, USA) according to the manufacturer's instructions. Targeted mutation analysis was performed for the specific mutations using polymerase chain reaction (PCR) and direct Sanger sequencing [13]. The coding exons and the flanking introns of the diseasecausing genes were amplified by PCR. Amplified PCR products were checked on agarose gel and purified using MultiScreen®HTS Millipore vacuum manifold (Millipore, Burlington, MA, USA). Thereafter, sequence analysis of the gene-specific PCR products was performed using the SeqMan 6.1 module of the Lasergene (DNA Star Inc., Madison, WI, USA) software package. Finally, the results of these sequence analyses were compared to the reference GenBank sequences for each gene. The diagnosis was confirmed by genetic targeted mutation analysis after birth.

Median maternal age, parity, gestational week of the IPNDT, prenatal test indications (mothers being carriers of the disease or at least one child with OA in their families), OA types, method of IPNDT (CVS or AC), IPNDT results (healthy or disease positive) and gestational outcomes (termination or continuation of pregnancy) after the diagnosis were evaluated.

Statistical analyses were conducted using the Statistical Package for the Social Sciences (SPSS®) version 22 software (https://www.ibm.com/SPSS-Statistics/software). Collected data are presented as median (range) values. Categorical data are presented as percentages. The study protocol was approved by the Hacettepe University Ethics Committee [\#GO 16/690].

\section{RESULTS}

Prenatal diagnosis was performed for three types of OAs: maple syrup urine disease (MSUD) $(n=5,50.0 \%)$, methylmalonic acidemia (MMA) $(n=3,30.0 \%)$ and isovaleric acidemia (IVA) $(n=2,20.0 \%)$. Three mutations in the $B C K D H A$ gene (c.347A $>\mathrm{G}, \mathrm{c} .905 \mathrm{~A}>\mathrm{C}$ and c.940C $>\mathrm{T}$ ) and two mutations in the $B C K D H B$ gene (c.502C $>\mathrm{T}$ and c.574G $>A$ ) were detected in the families with MSUD, who were all associated with the classic phenotype. All of the families with MMA had mutations in the MUT gene (c. $278 \mathrm{G}>\mathrm{A}$, c. $329 \mathrm{~A}>\mathrm{G}$ and c. $2200 \mathrm{C}>\mathrm{T}$ ). Finally, all of the families with IVA had mutations in the $I V D$ gene (c. 932C $>$ T for both). 
Demographic features and clinical characteristics of the cases are shown in Table 1. Median gestational week for IPNDT was 11.5 weeks. Ninety percent of the IPNDT indications was the presence of at least one child with OA in their families. Seventy percent of the IPNDTs were CVS. Two pregnancies (20.0\%) with disease-positive fetuses (one case with IVA, c.932C $>\mathrm{T}$ at the13th gestational week, and one case with MMA, c.278G $>$ A at the 19th gestational week) that were terminated after the parents opted to have IPNDT and decided to terminate the pregnancy in a case of OA. anemia can develop in infancy. Isovaleric acidemias are caused by a deficiency of isovaleryl-CoA dehydrogenase, the enzyme that converts isovaleryl-CoA to 3-methylcrotonyl CoA in the breakdown pathway of leucine. The accumulation of isovaleric acid in urine leads to a specific odor described as "sweaty feet." Vomiting, ketoacidosis, lethargy and coma may be observed depending on the severity of the disease [15].

There is usually no sign of OAs during the followup of the pregnancy. Family history of consanguineous

Table 1. Demographic features and clinical characteristic of the cases.

\begin{tabular}{|l|c|}
\hline Maternal age (median, range) & $30(21-35)$ \\
\hline Parity (median, range) & $1(0.4)$ \\
\hline Gestational week of invasive prenatal diagnostic test (median, range) & $11.5(11.0-17.0)$ \\
\hline $\begin{array}{l}\text { Invasive prenatal diagnostic test indications }(n, \%): \\
\text { mothers being carriers of the disease } \\
\text { at least one child with organic acidemia in their families }\end{array}$ & $1(10.0)$ \\
\hline $\begin{array}{l}\text { Organic acidemia types }(n, \%): \\
\text { maple syrup urine disease }\end{array}$ & $9(90.0)$ \\
\hline methylmalonic acidemia & $5(50.0)$ \\
isovaleric acidemia & $3(30.0)$ \\
\hline $\begin{array}{l}\text { Method of invasive prenatal diagnostic test }(n, \%): \\
\text { chorionic villi sampling }\end{array}$ & $2(20.0)$ \\
\hline amniocentesis & $7(70.0)$ \\
\hline Invasive prenatal diagnostic test results $(n, \%):$ & $3(30.0)$ \\
\hline healthy & $8(80.0)$ \\
disease positive & $2(20.0)$ \\
\hline Gestational outcome: & $2(20.0)$ \\
\hline termination of pregnancy & $8(80.0)$ \\
\hline continuation of pregnancy & \\
\hline
\end{tabular}

"One case with isovaleric acidemia and one case with methylmalonic acidemia.

\section{DISCUSSION}

Prenatal diagnostic procedures were performed for MSUD, MMA and IVA in this study. Maple syrup urine disease is an OA caused by a deficiency of branched-chain $\alpha$-ketoacid dehydrogenase complex, the second enzyme of the metabolic pathway of the three branched-chain amino acids, leucine, isoleucine and valine [14]. Psychomotor delay, feeding problems, and a maple syrup odor of the urine are the main characteristics of this disease [14]. Methylmalonic acidemia results from impaired metabolism of methylmalonic acid that is generated during the metabolism of certain amino acids (isoleucine, methionine, threonine or valine) and odd-chain fatty acids. Lethargy, seizures, muscular hypotonia and hypoglycemia are observed in neonates with MMA mut(0) or MMA mut(-) during an episode of metabolic decompensation. Microcephaly, pig-mentary retinopathy, nystagmus, secondary reduced visual acuity, hydrocephalus or megaloblastic marriage and/or siblings who died during the neonatal period may evoke suspicion for clinicians [16]. Unfortunately, there is no curative treatment for OAs at this time $[6,7]$. Treatment of metabolic decompensation periods, dietary management and application of medications such as L-carnitine, multivitamins and carglumic acid are the main conservative management protocols for OAs [6,7]. Thus, neonatal screening by tandem mass spectrometry in high-risk populations may be beneficial in order to detect cases at earlier stages of life and to take precautions for preventing life-threatening complications $[17,18]$. On the other hand, there are still ongoing debates about the ethical and cost-effective issues related to the newborn screening programs for OAs $[9,19]$. Therefore, establishing the most appropriate screening programs based on the specific characteristics of the populations seems to be the logical approach.

Prenatal diagnosis of inherited metabolic diseases is crucial, especially in countries with higher rates of con- 
sanguineous marriages due to the autosomal recessive pattern for the majority of the diseases [1]. According to the Turkish Demographic and Health Surveys, the rate of consanguineous marriage was found to be between 22.0$24.0 \%$ in Turkey [20]. Thus, inherited metabolic diseases are still an important healthcare problem for our country [20]. Although, current studies have reported promising results with cell-free DNA, IPDTs are still regarded as the gold standard method for the PND of OAs [21,22]. Amniocentesis, CVS and cordocentesis can all be performed with different procedure-related adventages and disadventages. Chorion villus sampling provides PND at earlier weeks of gestation but it has a procedure related fetal loss rate of $0.7-1.3 \%$, which was slightly higher than the rates reported for amniocentesis [23]. Additionally, CVS may sometimes lead to diagnostic uncertainty due to placental mosaicism [24]. Cordocentesis was reported to be associated with fetal-loss rates of 5.0-6.0\% [25]. Therefore, it is not routinely performed for PND of OAs $[21,22]$. Chorion villus sampling was the most common $(70.0 \%)$ IPDT procedure in this study and median gestational week for the IPDTs was 11.5 weeks. Two prenatally diagnosed cases with organic acidemia (one case with IVA and one case with MMA) were terminated after appropriate counseling. As PND of the case with IVA was performed by CVS, it gave us the opportunity for termination of pregnancy at an earlier gestational week compared to the case with MMA, which was diagnosed by amniocentesis. Earlier diagnosis of OAs facilitated the appropriate management of pregnancy in our institution.

Targeted mutation analysis was performed for all cases in this study. Although, metabolite studies and enzymatic assays are the other alternative diagnostic approaches, targeted mutation analysis has become the preferred method due to higher rates of sensitivity and specifity $[14,22]$. Mutation analysis in fetal DNA is a method of choice also in guidelines for MMA/PA [26]. Moreover, targeted mutation analysis gives the physician the opportunity for carrier screening that results in more effective preconceptional counseling $[14,22]$. Preimplantation genetics diagnosis may be an appropriate clinical option for couples with known mutations for OAs [27].

In conclusion, PND of OAs is critical. Appropriate prenatal counseling should be provided to families with known risk factors.

\section{ACKNOWLEDGMENTS}

We would like to extend our special thanks to all the medical staff at the Division of Perinatology, Hacettepe University Hospital, Ankara, Turkey for their efforts in the management of high-risk pregnancies.
Declaration of Interest. The authors report no conflicts of interest. The authors alone are responsible for the content and writing of this article.

\section{REFERENCES}

1. Chapman KA. Systemic organic acidemias: Identification, diagnosis, management and long term complications. J Pediatr Biochem. 2014; 4(4): 193-200.

2. Applegarth DA, Toone JR. Incidence of inborn errors of metabolism in British Columbia, 1969-1996. Pediatrics. 2000; 105(1): el0-e10.

3. Sanderson S, Green A, Preece M, Burton H. The incidence of inherited metabolic disorders in the West Midlands, UK. Arch Dis Child. 2006; 91(11): 896-899.

4. Wannmacher CM, Wajner M, Giugliani R, Giugliani ER, Costa MG, Giugliani MCK. Detection of metabolic disorders among high-risk patients. Rev Bras Genet. 1982;5(1):187-194.

5. Vaidyanathan K, Narayanan MP, Vasudevan DM. Organic acidurias: An updated review. Indian J Clin Bio-chem. 2011; 26(4): 319-325.

6. Morris A, Leonard J. Early recognition of metabolic decompensation. Arch Dis Child. 1997; 76(6): 555-556.

7. Dixon MA, Leonard JV. Intercurrent illness in inborn errors of intermediary metabolism. Arch Dis Child. 1992; 67(11): 1387-1391.

8. Rashed MS, Rahbeeni Z, Ozand PT. Application of electrospray tandem mass spectrometry to neonatal screening. Semin Perinatol 1999; 23(2): 183-193.

9. Dionisi Vici C, Deodato F, Röschinger W, Rhead W, Wilcken B. 'Classical'organic acidurias, propionic aciduria, methylmalonic aciduria and isovaleric aciduria: Long term outcome and effects of expanded newborn screening using tandem mass spectrometry. J Inherited Metab Dis. 2006; 29(2-3): 383-389.

10. Wajner M, de Moura Coelho D, Ingrassia $\mathrm{R}$, de Oliveira AB, Busanello ENB, Raymond $\mathrm{K}$, et al. Selective screening for organic acidemias by urine organic acid GC-MS analysis in Brazil: Fifteen-year experience. Clin Chim Acta. 2009; 400(1-2): 77-81.

11. Ramsay J, Morton J, Norris M, Kanungo S. Organic acid disorders. Ann Translational Med. 2018; 6(24): 472.

12. Vianey-Saban C, Acquaviva C, Cheillan D, Collardeau-Frachon S, Guibaud L, Pagan C, et al. Antenatal manifestations of inborn errors of metabolism: biological diagnosis. J Inherited Metab Dis. 2016; 39(5): 611-624. 
13. Sanger F, Nicklen $S$, Coulson AR. DNA sequencing with chain-terminating inhibitors. Proc Nat Acad Sci USA. 1977; 74(12): 5463-5467.

14. Morton DH, Strauss KA, Robinson DL, Puffen-berger EG, Kelley RI. Diagnosis and treatment of maple syrup disease: A study of 36 patients. Pediatrics. 2002; 109(6): 999-1008.

15. Schlune A, Riederer A, Mayatepek E, Ensenauer R. Aspects of newborn screening in isovaleric acidemia. Int J Neonat Screen. 2018; 4(1): 7-19.

16. Leonard J, Morris A. Inborn errors of metabolism around time of birth. Lancet. 2000; 356(9229): 583-587.

17. Shibata N, Hasegawa Y, Yamada K, Kobayashi H, Purevsuren J, Yang $\mathrm{Y}$, et al. Diversity in the incidence and spectrum of organic acidemias, fatty acid oxidation disorders, and amino acid disorders in Asian countries: Selective screening vs. expanded newborn screening. Mol Genet Metab Rep. 2018; 16: 5-10.

18. Vargas CR, Ribas GS, da Silva JM, Sitta A, Deon M Coelho DM, et al. Selective screening of fatty acids oxidation defects and organic acidemias by liquid chromatography/tandem mass spectrometry acylcarnitine analysis in Brazilian patients. Arch Med Res. 2018; 49(3): 205-212.

19. Hori D, Hasegawa Y, Kimura M, Yang Y, Verma IC, Yamaguchi S. Clinical onset and prognosis of Asian children with organic acidemias, as detected by analysis of urinary organic acids using GC/MS, instead of mass screening. Brain Develop. 2005; 27(1): 39-45.

20. Koc I, Eryurt MA. The causal relationship between consanguineous marriages and infant mortality in Turkey. J Biosocial Sci. 2017; 49(4): 536-555.
21. Chen C, Han L, Guo F, Ye J, Peng Z, Qiu W, et al. Noninvasive prenatal test of methylmalonic academia cblC type through targeted sequencing of cell-free DNA in maternal plasma. bioRxiv. 2018:425918.

22. Pérez Cerdá C, Pérez B, Merinero B, Desviat L, Pombo PR, Ugarte M. Prenatal diagnosis of propionic acidemia. Prenat Diagn. 2004; 24(12): 962-964.

23. Mujezinovic F, Alfirevic Z. Procedure-related complications of amniocentesis and chorionic villous sampling: A systematic review. Obstet Gynecol. 2007; 110(3): 687-694.

24. Alfirevic Z, Navaratnam K, Mujezinovic F. Amniocentesis and chorionic villus sampling for prenatal diagnosis. Cochrane Database Systematic Rev. 2017; 9. Art. No.: CD003252. doi: 10.1002/14651858. CD003252.pub2.

25. Antsaklis AI, Papantoniou NE, Mesogitis SA, Koutra PT, Vintzileos AM, Aravantinos DI. Cardiocen-tesis: An alternative method of fetal blood sampling for the prenatal diagnosis of hemoglobinopathies. Obstet Gynecol. 1992; 79(4): 630-633.

26. Baumgartner MR, Hörster F, Dionisi-Vici C, Haliloglu G, Karall D, Chapman KA, et al. Proposed guidelines for the diagnosis and management of methyl-malonic and propionic acidemia. Orphanet J Rare Dis. 2014; 9(1): 130-166.

27. Alberola TM, Bautista-Llácer R, Vendrell X, GarcíaMengual E, Pardo M, Vila M, et al. Case report: Birth of healthy twins after preimplantation genetic diagnosis of propionic acidemia. J Assist Reprod Genet. 2011; 28(3): 211-216. 